University of Wollongong

Research Online

Faculty of Social Sciences - Papers (Archive) Faculty of Arts, Social Sciences \& Humanities

$1-1-2017$

\title{
Time2bHealthy - An online childhood obesity prevention program for preschool-aged children: A randomised controlled trial protocol
}

Megan Hammersley

University of Wollongong, mlh965@uowmail.edu.au

Rachel A. Jones

University of Wollongong, rachelj@uow.edu.au

Anthony D. Okely

University of Wollongong, tokely@uow.edu.au

Follow this and additional works at: https://ro.uow.edu.au/sspapers

Part of the Education Commons, and the Social and Behavioral Sciences Commons

Research Online is the open access institutional repository for the University of Wollongong. For further information contact the UOW Library: research-pubs@uow.edu.au 


\title{
Time2bHealthy - An online childhood obesity prevention program for preschool- aged children: A randomised controlled trial protocol
}

\author{
Abstract \\ Background The use of parent-focused internet-based programs for the prevention and treatment of \\ childhood obesity has shown promise but there is an acknowledged gap in parent-focused interventions \\ which target the early childhood stage. Methods The aim of this study is to determine the efficacy of \\ Time2bHealthy - an online healthy lifestyle program for parents of preschool-aged children. The program \\ will be evaluated using a two-arm, parallel, randomised controlled design. The 11-week program is \\ underpinned by Social Cognitive Theory and consists of interactive modules on healthy eating, physical \\ activity, screen-time and sleep. The intervention also involves elements of social media, where \\ participants share discuss ideas and experiences and they can interact and obtain information with \\ experts. Time $2 \mathrm{bHealthy}$ is being compared to a comparison condition. Outcomes include change in BMI \\ (primary outcome), dietary intake, physical activity, sleep, child feeding, parental role-modelling and parent \\ self-efficacy. Process evaluation data, such as adherence and engagement with the online forums, will \\ also be collected. Discussion Time2bHealthy is the first randomised controlled trial to our knowledge to \\ assess the efficacy of an online parent-focussed healthy lifestyle program for preschool-aged children in \\ changing body mass index. Early childhood is a crucial time for establishing healthy lifestyle behaviours \\ and parents play an important role. This study therefore fills an important gap in the literature. If found to \\ be efficacious, Time2bHealthy has potential for broad-reach access and translation into primary health \\ care services. Trial Registration The study is registered with the Australian and New Zealand Clinical \\ Trials Registry (12616000119493). \\ Disciplines \\ Education | Social and Behavioral Sciences

\section{Publication Details} \\ Hammersley, M. L., Jones, R. A. \& Okely, A. D. (2017). Time2bHealthy - An online childhood obesity \\ prevention program for preschool-aged children: A randomised controlled trial protocol. Contemporary \\ Clinical Trials, 61 73-80.
}




\title{
Time2bHealthy - an online childhood obesity prevention program for preschool-aged children: a randomised controlled trial protocol
}

\begin{abstract}
Background

The use of parent-focused internet-based programs for the prevention and treatment of childhood obesity has shown promise but there is an acknowledged gap in parent-focused interventions which target the early childhood stage.
\end{abstract}

\section{Methods}

The aim of this study is to determine the efficacy of Time2bHealthy - an online healthy lifestyle program for parents of preschool-aged children. The program will be evaluated using a two-arm, parallel, randomised controlled design. The 11-week program is underpinned by Social Cognitive Theory and consists of interactive modules on healthy eating, physical activity, screen-time and sleep. The intervention also involves elements of social media, where participants share discuss ideas and experiences and they can interact and obtain information with experts. Time $2 b H e a l t h y$ is being compared to a comparison condition. Outcomes include change in BMI (primary outcome), dietary intake, physical activity, sleep, child feeding, parental role-modelling and parent self-efficacy. Process evaluation data, such as adherence and engagement with the online forums, will also be collected.

\section{Discussion}

Time2bHealthy is the first randomised controlled trial to our knowledge to assess the efficacy of an online parent-focussed healthy lifestyle program for preschool-aged children in changing body mass index. Early childhood is a crucial time for establishing healthy lifestyle 
behaviours and parents play an important role. This study therefore fills an important gap in the literature. If found to be efficacious, Time2bHealthy has potential for broad-reach access and translation into primary health care services.

\section{Trial Registration}

The study is registered with the Australian and New Zealand Clinical Trials Registry (12616000119493).

\section{Keywords}

Childhood obesity, eHealth, internet, online, parent 


\section{Introduction}

Overweight and obesity in early childhood is associated with a range of short- and long-term health consequences. Furthermore, overweight children have at least twice the risk of remaining overweight into their adult life compared to children in the healthy weight range [1]. Effective weight management interventions can reduce the likelihood of childhood overweight and obesity continuing into adulthood. Increasing physical activity and improving eating behaviours are recognised cornerstone weight management strategies [1,2]. There is also increasing evidence regarding the importance of limiting screen-time [3], reducing sedentary activities $[4,5,6]$ and maintaining healthy sleeping patterns $[7,8,9]$.

Parental influence and role-modelling play a key part in the development of such behaviours $[10,11,12]$. Therefore, the role of parents in overweight and obesity prevention and intervention programs is critical. Previous reviews have highlighted the success of interventions which involve parents compared to those that do not $[13,14,15]$. This is particularly true for programs that target young children. Despite the importance of parental involvement in overweight and obesity prevention and treatment programs, there are recognised barriers such as scheduling of appointments/ sessions [15], stigma, parental denial [16], childcare for other siblings [17], travel [18] and cost [15] that prevent parental involvement and potentially success of the programs.

The use of the online medium for overweight and obesity prevention and treatment programs offers advantages compared to face-to-face programs in convenience and accessibility. There have been an increasing number of online healthy lifestyle interventions for children in recent years. Systematic reviews have demonstrated that such interventions are efficacious in improving obesity-related behaviours and are cost effective, however the majority of studies have been conducted in the primary- or high-school age groups and most have not involved 
parents as an agent of change [19,20,21]. A recent meta-analysis of parent-focussed eHealth obesity interventions found that while there was no significant effect found in BMI/BMI zscore change, half of the studies demonstrated significant improvements obesity-related behaviours such as diet or physical activity compared to a control group. In this review there was an absence of studies which included children under the age of five years, an age group where parental influence is probably more profound than older childhood and adolescence [12]. It was therefore recommended that larger, high quality studies are conducted which transform successful components from face-to-face interventions into an eHealth format, particularly those which target younger age groups and focus on parents as agents of change [19]. The aim of this paper is to describe the protocol for the Time2bHealthy Study. The purpose of the study is to determine the efficacy of the parent-focused Time2bHealthy online program in facilitating behaviour change among preschool-aged children who are overweight, or at risk of becoming overweight. The primary hypothesis is that children in the intervention group will demonstrate significantly greater reductions in BMI/BMI z-score compared to the comparison group by the 6-month follow-up. Secondary outcomes including child dietary intake, physical activity, screen-time, sleep and parent self-efficacy will also be assessed.

\section{Methods}

\section{Study Design}

The Time2bHealthy study is a two-arm randomised controlled trial involving parent-child dyads (Figure 1). Time2bHealthy is based on formative research with parents of preschoolaged children. This research analysed the content of 300 publicly available websites containing healthy lifestyle information for children of preschool age and found that the websites lacked strategies on how to practically apply the information and set goals to assist 
in changing behaviours. Focus group results from this research similarly indicated that parents would find information of how to implement changes helpful. Parents also advised that an online program would need to be easy to use and be flexible and highly credible. Personalised feedback from a health professional was also important to parents [22].

Based on the results of this research, a 10-week, five-module online program Time2bHealthy - was developed which covered the areas of healthy meals, healthy snacks and drinks, physical activity and screen-time. The program was piloted with 47 dyads and was found to be acceptable, potentially efficacious and had a high level of retention [23] and a randomised controlled trial is now required to fully test the efficacy of the intervention. Due to more recent research into the effects of sleep on overweight and obesity in children, an additional module on sleep was added to the program for the current trial. Content of the modules was also updated according to the latest evidence-based recommendations $[24,25,26]$ and the behaviour change and goal-setting aspects of the program were strengthened.

The study will be reported according to the Consolidated Standards of Reporting Trials (CONSORT) statement [27]. The study has been approved by the University of Wollongong Human Research Ethics Committee (HE15/354) and is registered with the Australian and New Zealand Clinical Trials Registry (12616000119493).

\section{Participant Recruitment and Eligibility Criteria}

Participants are being recruited from the Illawarra, Southern and South-Western Sydney, Southern Highlands and Shoalhaven areas of New South Wales and Melbourne, Victoria. To assist with recruitment, organisations and individuals such as early childhood education 
centres, schools, playgroups, general practices, early childhood nurses, preschool swimming and sporting activities are being contacted and asked to distribute flyers and/or display posters. Articles have been placed in university and local health district newsletters, a Facebook page was created to communicate information about the study throughout the recruitment areas and a media release was sent to media agencies.

Potential participants are provided with a participant information sheet and screened for eligibility via phone or email. Participants are eligible if they live in one of the geographical areas described, the child is 2-5 years of age and not yet attending school at the time of recruitment and the child is at or above the WHO $50^{\text {th }}$ percentile for body mass index for their age and sex. Parents are also required to have a Facebook account or be willing to create one for the duration of the study.

Child participants are excluded if they are taking medications or have a medical condition that can affect weight. As such, children are excluded if they are taking any of the following medications: Ritalin or other therapy for attention deficit hyperactivity disorder, long-term steroids, anti-psychotic medication. Furthermore, children are excluded if they have any of the following conditions or disabilities; Prader-Willi Syndrome, Bardet-Biedl Syndrome, Diabetes, Phenylketonuria or other metabolic disorders, Cystic Fibrosis, significant physical or developmental disability (that restricts age-appropriate play) or other conditions associated with overweight/obesity. Children with conditions such as Coeliac Disease or food allergies are able to participate, but parents are informed that some of the healthy eating content of the program will not be entirely appropriate and they will need make their own modifications to some of the information provided to suit their child's specific dietary requirements.

Eligible participants provide informed consent after reading the participant information sheet by completing a written consent form. Eligibility is confirmed at the baseline data collection 
visit when child height and weight are measured and body mass index is calculated to determine if the child is at or above the $\mathrm{WHO} 50^{\text {th }}$ percentile for age and sex. Recruitment commenced in January 2016, with participants being recruited into six cohorts on a rolling basis. It is anticipated that recruitment will be completed by June 2017 .

\section{Power and Sample Size}

We expect an effect size of approximately 0.4 for this trial (based on the results of the pilot study). To detect a statistically significant difference between groups (alpha=0.05 and power=0.8), 136 participants are required (68 per group) and considering an estimated attrition rate of $15 \%, 160$ participants are planned to be recruited ( 80 per group).

\section{Randomisation}

Once participants are recruited and baseline measures are collected for each cohort, participants are randomised into the intervention or comparison group. Randomisation is conducted by a data manager using a concealed computerised random number generator. The data manager is not involved in the recruitment or delivery of the intervention. Results of the randomisation are then communicated to the researcher responsible for implementing the intervention. Height, weight, physical activity, dietary intake, sleep, screen-time, parental modelling and self-efficacy are assessed at baseline, post-intervention and 6-month followup. Baseline data collection is completed prior to randomisation, so data collectors are blinded to group allocation. At post-intervention and 6-month follow-up data collection timepoints accelerometers are fitted and questions on dietary intake, sleep, screen-time, rolemodelling and parent self-efficacy are entered directly into an iPad by the participants. Height and weight measurements at the follow-up time-points are taken by data collectors who are blinded to group allocation.

\section{Theoretical Framework}


The intervention is guided by Bandura's Social Cognitive Theory, which proposes that there are three influences on behaviour: personal, behavioural and environmental (which is also known as reciprocal determinism) [28]. The interaction of the personal, environmental and behavioural influences within the Time2bHealthy intervention are illustrated in Figure 2.

- Personal influence refers to an individual's self-efficacy (or their personal belief in their ability) to carry out a behaviour. This will be based on their personality, knowledge, beliefs, self-perceptions and expectations. Knowledge and beliefs of the importance of healthy eating, physical activity, sleep and limiting screen-time for preschool-aged children are addressed through the program content of the modules.

- Environmental influence refers to supportive environments which assist an individual to carry out a behaviour. An individual will be influenced by physical and societal influences in the environment. Videos demonstrating effective use of skills and behaviours by others will provide participants with vicarious learning. Communication, feedback and reinforcement from other participants via the Facebook group and from research staff via individualised communication also assists in supporting participants in practising the skills and behaviours.

- Behavioural influence refers to the response by the individual once they have practised carrying out a behaviour. This experience will determine how often and how well they will carry out a behaviour. After setting SMART goals specific, measureable, achievable, realistic and time-framed) [29] and action plans, participants will practice the skills and behaviours. Positive reinforcement will be gained through the monitoring of progress with their goals and action plans and the personal benefits experienced.

The intervention addresses the four key processes of Social Cognitive Theory for learning and adapting new behaviours: attention, retention, production and motivation [28]. Attention 
is addressed by ensuring that the website is easy to use and contains interactive evidencebased components (videos, activities and goal-setting), so participants are engaged.

Retention is supported through an optimal length for the program, interaction with other participants through the closed Facebook group and quizzes to support parents in remembering the key content from each module. Production is addressed through goalsetting, action planning, addressing barriers and behaviour rehearsal. Motivation is addressed through creating cognitive dissonance by parents documenting current behaviours (e.g. in the activity planner) and asking parents to identify the positive outcomes and expectations as a result of performing the planned actions. Throughout the goal-setting process, parents are asked their motivation to make a change and SMART goals [29] are set, where parents are asked to aim challenging, yet realistic goals.

\section{Intervention Development}

A backwards intervention mapping process was utilised in designing the study to align the Time2bHealthy intervention activities to the theory and target behaviours (developed by Robinson [30,31]). This process involved determining the overall goal first and then working backwards to identify the major and sub-categories, the target behaviours needed to achieve these and strategies based around the elements of Social Cognitive Theory that can be applied to support the theory. Figure 3 illustrates this process for the physical activity component of the intervention.

Table 1 outlines the components of each of the modules of the Time2bHealthy online program. The content is based on evidence-based guidelines and recommendations for dietary intake, physical activity, screen-time and sleep.

\section{Time2bHealthy Intervention}


Time 2 bHealthy consists of 6 modules on topics including nutrition $(\mathrm{n}=2)$, physical activity, screen-time and sleep and is delivered over a period of 11 weeks. Each module takes approximately 30 minutes to complete and modules are completed over a two-week period. The modules involve participants reading text on each topic, watching videos, completing activities and setting goals. Goal-setting and subsequent revision of goals has been demonstrated to be important in the success of lifestyle behaviour change interventions $[23,32,33]$. Feedback is provided by a dietitian via the website regarding the goals set, with advice provided to enhance the goals in line with the SMART goal framework [29]. Additionally, participants receive regular emails to remind them to log on to the website, aimed to assist with participant retention. A closed (secret) Facebook group is accessible, where participants have the opportunity to communicate with other members of the cohort as well as the dietitian. The Facebook groups are monitored daily and moderated by the dietitian to ensure that the information discussed is consistent with evidence-based guidelines. Participants are asked to provide regular input to these groups by sharing healthy recipes/photos of meals, healthy snacks, suitable physical activities and personal ideas and experiences in regard to reducing screen-time and improving sleep. Incentives (either shopping gift cards or vouchers to a children's museum) are provided in return for the time taken for participants to contribute this input.

At the end of the online program at 11-weeks, participants continue to receive fortnightly contact via email. These emails contain infographics which provide a summary of the content from the online program and direct participants to visit the online program to review material and their progress with goals set.

\section{Comparison Condition}


Participants assigned to the comparison group receive fortnightly emails directing them to various topics on the evidence-based, Australian government-funded parenting website: Raising Children Network. The topics provided are of a similar nature to the intervention group (nutrition, physical activity, screen-time and sleep) and other general health topics relevant to the preschool life stage. The comparison group do not have access to any interactive components such as practical activities, goal setting, and individualised feedback. Additionally, the comparison group are also offered access to the Time2bHealthy program at the completion of the follow-up data collection; however, due to time constraints participants will not have access to the Facebook group or receive the regular email contact when they have access to the Time2bHealthy program.

\section{Outcome Measures}

All measures are collected via face-to-face appointments at the University of Wollongong Early Start building, the participant's home or a community setting, where both parent and child attend. All questionnaires are completed by parents using an iPad. Appointments are approximately 30-45 minutes in duration. The outcome measures collected are described in table 2 .

\section{Primary outcome measure}

Body Mass Index is calculated with height and weight measurements. Height and weight are measured using a standardised method [2]. Height is measured to the nearest $0.1 \mathrm{~mm}$ using a stadiometer. Weight is measured to the nearest $0.1 \mathrm{~kg}$ using a SECA scale. Height and weight are measured twice and recorded. An average of the two measurements are then used for BMI calculations. In instances where height measurements differ more than $0.5 \mathrm{~cm}$ and weight measurements differ more than $0.5 \mathrm{~kg}$, a third measurement is taken. 


\section{Physical Activity}

Actigraph GT3X+ accelerometer (ActiGraph Corporation, Pensacola, FL) are used to measure the intensity and amount of physical activity that is occurring over time. The Actigraph GT3X+ accelerometer is a small, light-weight device which records tri-axial movement (up and down, side to side and forward and backward). Accelerometers have been extensively used in physical activity studies in children and they have been validated for use in the preschool age-group [34].

Accelerometers collect very high-frequency raw data $(30 \mathrm{~Hz})$ on activity counts, which are stored as epochs in the device and then downloaded for analysis. Cut-points to differentiate physical activity intensity that are appropriate for the preschool age-group will be utilised in the analysis $[35,36]$.

All child participants wear an Actigraph accelerometer around the waist on an elasticised belt continuously for a period of seven days (for 24 hours per day), removing them only for a bath/shower or water activities. The accelerometers are fitted to participants at the time of the face-to-face appointments and they are collected from the participant's home or participants return them in a reply paid envelope. Parents are provided with instructions on how to remove and re-fit the device.

Sleep

Accelerometers will be used to assess sleep habits in conjunction with a questionnaire [37]. A number of recent studies have utilised accelerometers in children for a 24-hour period to assess both sleep and physical activity [38-41]. 
The questionnaire used consists of eight questions and has been modified from a tool which has been previously validated in the preschool age group (Children's Sleep Habits Questionnaire [37]) and includes questions about typical bedtime and wake up time, typical time and duration of daytime nap and other sleep habits. The information from this questionnaire will be utilised in conjunction with accelerometer data to determine sleep duration, a method that has been used in other studies $[42,43]$.

\section{Dietary Intake}

Two methods are used to assess dietary intake. Parents firstly complete four multicomponent questions which have been modified from a parent-reported questionnaire which has been validated in the preschool age group (The Eating and Physical Activity Questionnaire (EPAQ)) [44]. The second method is a 24-hour recall of the previous day's dietary intake. Parents enter this information into the 'Easy Diet Diary' app (Xyris Software (Australia) Pty Ltd) on an iPad.

\section{Child Feeding}

The questionnaire consists of 12 questions from the validated Child Feeding Questionnaire [45] and asks parents about their attitudes, beliefs and practices regarding child feeding.

\section{Screen-time}

This questionnaire asks parents to estimate the usual amount of screen-time for their child on a typical weekday and weekend day to determine average screen-time per week. Questions are also included about the availability of screens and rules about screen entertainment. The questions are based on a questionnaire previously assessed for reliability [46,47].

\section{Parental Modelling}


Parents are asked about parental modelling of the behaviours addressed in the intervention (nutrition, physical activity, screen-time and sleep). As there is not an appropriate validated tool in the existing literature, these four questions have been developed after reviewing other parent-modelling questionnaires such as the Parental Modelling of Eating Behaviours Scale [48] and the Home Environment Survey [49].

\section{Parent self-efficacy}

Parents are asked about their self-efficacy relating to their child's nutrition, physical activity, screen-time and sleep. This questionnaire consists of 13 questions and has been modified from a previously validated questionnaire [50].

\section{Demographic Characteristics}

Demographic information is collected from the parents via an iPad. Variables include participant child age, child sex, child DOB, parent age, parent sex, parent height and weight, Aboriginal or Torres Strait Islander status, language spoken at home, postcode, where they found out about the study, child care/preschool attendance, number of children in household, number of adults in household, marital status, highest level of education, family income, relationship to child, birth weight, premature birth and duration of breastfeeding. This information is collected at the baseline time-point only.

\section{Process Evaluation}

Process evaluation is being conducted via a questionnaire which participants are asked to complete at the end of the online program. Participants are asked to complete a series of questions with responses on a Likert scale. Specifically, participants are asked about program content, if the content was interesting, easy to understand, relevant and appropriate. They are asked about the length of the program and duration of the modules, the goal-setting component and feedback received from the dietitian. Participants are asked if they completed 
the modules in one sitting or at different times and how much time they spent to complete each module. They are asked about the online delivery of the program and if they would have preferred a different format. They are also asked about the Facebook component of the program.

\section{Statistical Analysis}

The outcomes will be assessed by comparing the differences in change over time between the intervention and comparison groups. Linear mixed or generalised linear mixed models will be used to analyse the data to determine differences between groups over time (baseline, 12 weeks and 6 months) with adjustment for potential covariates (see below).

Intention-to-treat principles will be used, with all participants analysed in the group which they are randomised. Covariates will include age, and accelerometer wear time.

Two types of exploratory analyses will be conducted to examine the theoretical assumptions of the intervention. First, hypothesised mediators of change in BMI (child physical activity, screen- time, eating behaviours, sleep, self-efficacy, parental role-modelling) will be examined using the PROCESS SPSS Macro version 2.16. Potential moderators of the intervention effects (e.g., child age and sex) will also be explored using the PROCESS SPSS Macro version 2.16 [51].

Relationships between demographic data and outcome variables will also be considered.

\section{Discussion}

This paper describes the Time2bHealthy study - the first randomised controlled trial to our knowledge to assess the efficacy of an online parent-focussed healthy lifestyle program for preschool-aged children in changing body mass index. This study therefore fills a gap in the literature and addresses many limitations in similar studies conducted in older age groups, 
such as risk of bias, small sample size, lack of follow-up and parents not being the agents of change in most studies [19].

The study has a number of strengths, including a planned sample size that has been calculated to ensure adequate statistical power. Objective and valid data collection methods are being used for outcome measures. A 6-month follow-up will determine if the changes made during the intervention can be maintained. The mode of delivery that is being used for the study has demonstrated the potential to be effective. Multiple obesity-related behaviours including healthy eating, physical activity, screen-time and sleep are targeted in the intervention. Finally, the intervention has been designed in line with Social Cognitive Theory using an established backwards intervention mapping process.

There are however some risks and limitations. The study design includes children who have a healthy weight and it is therefore possible that the BMI changes may be diluted. As the comparison group also receives information on similar topics, it is possible that behaviour change may occur in both groups. Due to the use of self-reported data for some measures, there is the potential for parents to intentionally or unintentionally misreport, however, this is an issue that is common to all free-living studies assessing behavioural measures such as dietary intake. There is limited opportunity to establish rapport with participants (compared to face-to-face or telephone-based interventions). Initial rapport will be established during the initial face-to-face data collection appointment. A community will also be established online through the closed Facebook group to facilitate communication and building rapport with and between participants.

This study will make an important contribution to the literature on internet interventions for the prevention and/or treatment of childhood obesity where parents are the agent of change. Evidence indicates that carefully targeted internet childhood obesity treatment and prevention 
programs have promising potential and the internet is a mode of delivery which has been shown to offer specific appeal to the target group [22,23]. A large proportion of Australian households are connected to the internet (83\% in 2012-2013), including those in regional, rural and remote areas [52], potentially enabling widespread access to programs regardless of geographic location. The intervention also has the potential for broad reach as it negates many barriers associated with traditional delivery methods [15-18].

If found to be efficacious, the Time $2 b H e a l t h y$ study has potential for translation into primary health care services, in particular for parents in rural and remote areas, where access to obesity prevention and management services can be limited and overweight and obesity prevalence is higher [2].

\section{Declarations}

\section{Ethics approval and consent to participate}

The study protocol has been approved by the University of Wollongong Human Research Ethics Committee (HE15/354). Participants provide informed written consent before participating.

\section{Consent for publication}

Not applicable

\section{Availability of data and material}

The datasets from this study are available from the corresponding author on written request.

\section{Competing interests}

The authors declare that they have no competing interests.

\section{Funding}


This research has been conducted with the support of the Australian Government Research Training Program Scholarship. The study was also supported by funding from Australian Health Management. ADO is supported by a National Heart Foundation of Australia Career Development Fellowship (CR11S 6099). The funding body was not involved in the design, data collection, analysis, interpretation or writing.

Authors' contributions

MLH is a PhD student and managed the day-to-day running of the study, managed data collection, contributed to study design, program content and drafted and edited the manuscript. $\mathrm{RAJ}$ is $\mathrm{PhD}$ supervisor to $\mathrm{MH}$ and contributed to the study design, program content and edited the manuscript. $\mathrm{ADO}$ is $\mathrm{PhD}$ supervisor to $\mathrm{MH}$ and contributed to study design, program content and edited the manuscript. All authors read and approved the final manuscript.

\section{Acknowledgements}

The authors would like to thank Marijka Batterham for providing statistical support for this study. The authors would also like to thank the participants of the study and the many individuals, organisations who have assisted in communicating information about the study to families. 
References

1. Luttikhuis, H.O., Baur, L., Jansen, H., Shrewbury, V. A., O'Malley, C., Stolk, R. P., Summerbell, C. D. 2009. Interventions for treating obesity in children. Cochrane Database of Systematic Reviews.

2. National Health and Medical Research Council. 2013. Clinical Practice Guidelines for the Management of Overweight and Obesity in Adults, Adolescents and Children. Melbourne: National Health and Medical Research Council.

3. Hinkley, T., Salmon, J., Okely, A. D., Crawford, D., Hesketh, K. 2012b. Preschoolers' physical activity, screen time, and compliance with recommendations. Med Sci Sports Exerc 44:458-65.

4. Okely, A. D., Jones, R. A. 2011. Sedentary Behavior Recommendations for Early Childhood. In: Tremblay, R., Barr, R., Peters, R. \& Boivin, M. (eds.) Encyclopedia on Early Childhood Development. Montreal: Centre for Excellence for Early Childhood Development.

5. Reilly, J. J. 2008. Physical activity, sedentary behaviour and energy balance in the preschool child: opportunities for early obesity prevention. Proc Nutr Soc 67:317-25.

6. Okely, A. D., Salmon, J., Vella, S. A., Cliff, D., Timperio, A., Tremblay, M., Trost, S.G., Shilton, T., Hinkley, T., Ridgers, N., Phillipson, L., Hesketh, K., Parrish, A.-M., Janssen, X., Brown, M., Emmel, J., Marino, N. 2012. A Systematic Review to inform the Australian Sedentary Behaviour Guidelines for Children and Young People. Report prepared for the Australian Government Department of Health, June 2012.

7. Cappuccio, F., Taggart, F., Kandala, N., Currie, A., Peile, E., Strages, S., Miller, M. 2008. Meta-analysis of short sleep duration and obesity in children and adults. Sleep 31:619-626.

8. Fatima Y., Doi SA., Mamun AA. 2016. Sleep quality and obesity in young subjects: a meta-analysis. Obes Rev 17:1154-66.

9. Thind H., Davies SL., Lewis, T., Pekmezi D., Evans R., Baskin ML. 2014. Does short sleep lead to obesity among children and adolescents? Current understanding and implications. Am J Lifestyle Med 9:428-437.

10. Golley, R. K., Hendrie, G. A., Slater, A., Corsini, N. 2011. Interventions that involve parents to improve children's weight-related nutrition intake and activity patterns what nutrition and activity targets and behaviour change techniques are associated with intervention effectiveness? Obes Rev 12:114-130. doi: 10.1111/j.1467789X.2010.00745.x.

11. Niemeier, B. S., Hektner, J. M., Enger, K. B. 2012. Parent participation in weightrelated health interventions for children and adolescents: A systematic review and meta-analysis. Prev Med 55:3-13. doi: 10.1016/j.ypmed.2012.04.021. 
12. Natale, R. A., Messiah, S. E., Asfour, L., Uhlhorn, S. B., Delamater, A., Arheart, K. L. 2014. Role modeling as an early childhood obesity prevention strategy: Effect of parents and teachers on preschool children's healthy lifestyle habits. J Dev Behav Pediatr 35:378-387. doi: 10.1097/DBP.0000000000000074.

13. Ho, M., Garnett, S. P., Baur, L., Burrows, T., Stewart, L., Neve, M., Collins, C. 2012. Effectiveness of lifestyle interventions in child obesity: Systematic review with metaanalysis. Pediatrics 130, e1647-e1671. doi: 10.1542/peds.2012-1176.

14. Young, K. M., Northern, J. J., Lister, K. M., Drummond, J. A., O'Brien, W. H. 2007. A meta-analysis of family-behavioral weight-loss treatments for children. Clin Psychol Rev 27:240-9.

15. Grimes-Robison., C. Evans, R. R. 2008. Benefits and barriers to medically supervised pediatric weight-management programs. J Child Health Care 12:329-343. doi: $10.1177 / 1367493508096319$.

16. Kelleher, E., Davoren, M.P., Harrington, J.M., Shiely, F., Perry, I.J., McHugh, S.M. 2017. Barriers and facilitators to initial and continued attendance at community-based lifestyle programmes among families of overweight and obese children: a systematic review. Obes Rev 18(2):183-194. doi: 10.1111/obr.12478.

17. Warren, J. M., Golley, R. K., Collins, C. E., Okely, A. D., Jones, R. A., Morgan, P. J., Perry, R. A., Baur, L. A., Steele, J. R., Magarey, A. M. 2007. Randomised controlled trials in overweight children: Practicalities and realities. Int J Pediatr Obes, 2:73-85.

18. Fitch, A., Fox, C., Bauerly, K., Gross, A., Heim, C., Judge-Dietz, J., Kaufman, T., Krych, E., Kumar, S., Landin, D., Larson, J., Leslie, D., Martens, N., MonaghanBeery, N., Newell, T., O'Connor, P., Spaniol, A., Thomas, A., Webb, B. 2013. Prevention and Management of Obesity for Children and Adolescents. Institute for Clinical Systems Improvement.

19. Hammersley, M.L., Jones, R.A., Okely, A.D. Parent-focused childhood and adolescent overweight and obesity ehealth interventions: a systematic review and meta-analysis. J Med Internet Res 18:e203. doi: 10.2196/jmir.5893.

20. Nguyen, B., Kornman, K. P. \& Baur, L. A. 2011. A review of electronic interventions for prevention and treatment of overweight and obesity in young people. Obes Rev 12:e298-e314. doi: 10.1111/j.1467-789X.2010.00830.x.

21. An, J., Hayman, L., Park, Y., Dusaj, T., Ayres, C. 2009. Web-based weight management programs for children and adolescents: a systematic review of randomized controlled trial studies. ANS Adv Nurs Sci 32:222-240. doi: 10.1097/ANS.0b013e3181b0d6ef.

22. Jones, R. A., Price, N., Okely, A. D., Lockyer, L. 2009. Developing an online program to prevent obesity in preschool-aged children: What do parents recommend? Nutr Diet 66:151-157. 
23. Jones, R., Wells, M., Okely, A., Lockyer, L., Walton, K. 2011. Is an online healthy lifestyles program acceptable for parents of preschool children? Nutr Diet 68:149154.

24. National Health and Medical Research Council. 2013. Australian Dietary Guidelines. Canberra: National Health and Medical Research Council.

25. Department of Health. 2014. National Physical Activity Recommendations for Children (0-5 years). Canberra: Commonwealth of Australia, Department of Health.

26. Hirshkowitz, M., Whiton, K., Albert, S.M., Alessi, C., Bruni, O., DonCarlos, L., Hazen, N., Herman, J., Katz, E.S., Kheirandish-Gozal, L., Neubauer, D.N., O’Donnell, A.E., Ohayon,M., Peever, J., Rawding, R., Sachdeva, R.C., Setters, B., Vitiello, M.V.,Ware, J.C., Adams, P.J. 2015. National Sleep Foundation's sleep time duration recommendations: methodology and results summary. Sleep Health, 1:4043. doi: 10.1016/j.sleh.2014.12.010

27. Moher, D., Liberati, A., Tetzlaff, J. \& Altman D.G. The PRISMA Group. 2009. Preferred Reporting Items for Systematic Reviews and Meta-Analyses: The PRISMA Statement. PLoS Med 6:e1000097. doi: 10.1371/journal.pmed.1000097.

28. Bandura, A. 1986. Social Foundations of Though and Actions., NJ:Prentice Hall, Englewood Cliffs.

29. Locke, E. A., Latham, G. P. 2002. Building a practically useful theory of goal setting and task motivation: A 35-year odyssey. Am Psych 57:705-717.

30. Robinson, T.N., Borzekowski, D.L.G. 2006. Effects of the SMART Classroom Curriculum to Reduce Child and Family Screen Time. Journal of Communication 56:1-26.doi: 10.1111/j.1460-2466.2006.00001.x

31. Cornelius, M., Armel, K.C., Hoffman, K., Allen, L., Bryson, S.W., Desai, M., Robinson, T.N. 2014. Increasing energy- and greenhouse gas-saving behaviors among adolescents: a school-based cluster-randomized controlled trial. Energy Efficiency 7:217-242. doi: 10.1007/s12053-013-9219-5.

32. Estabrooks, P. A., Nelson, C. C., Xu, S., King, D., Bayliss, E. A., Gaglio, B., Nutting, P. A., Glasgow, R. E. 2005. The frequency and behavioral outcomes of goal choices in the self-management of diabetes. Diabetes Educator, 31:391-400.

33. Nothwehr, F., Yang, J. 2007. Goal setting frequency and the use of behavioral strategies related to diet and physical activity. Health Educ Res, 22:532-538.

34. National Health and Medical Research Council. 2013. Clinical Practice Guidelines for the Management of Overweight and Obesity in Adults, Adolescents and Children. Melbourne: National Health and Medical Research Council.

35. De Vries, S. I., Van Hirtum, H. W., Bakker, I., Hopman-Rock, M., Hirasing, R. A., Van Machelen, W. 2009. Validity and reproducibility of motion sensors in youth: a systematic update. Med Sci Sports Exerc 41:818-27. doi:

10.1249/MSS.0b013e31818e5819. 
36. Pate, R.R., Almeida, M.J., McIver, K.L., Pfeiffer, K.A., Dowda, M. 2006. Validation and calibration of an accelerometer in preschool children. Obesity 14:2000-2006. doi: 10.1038/oby.2006.234.

37. Sneddon, P., Peacock, G. G., Crowley, S. L. 2013. Assessment of sleep problems in preschool aged children: an adaptation of the children's sleep habits questionnaire. Behav Sleep Med 11:283-96. doi: 10.1080/15402002.2012.707158.

38. Janssen, X., Cliff, D.P., Reilly, J.J., Hinkley, T., Jones, R.A., Batterham, M., Ekelund, U., Brage, S., Okely, A.D. 2013. Predictive validity and classification accuracy of ActiGraph energy expenditure equations and cut-points in young children. PloS one 8:e79124. doi: 10.1371/journal.pone.0079124.

39. Kinder, J. R., Lee, K. A., Thompson, H., Hicks, K., Topp, K., Madsen, K. A. 2012. Validation of a hip-worn accelerometer in measuring sleep time in children. $J$ Pediatr Nurs 27:127-33. doi: 10.1016/j.pedn.2010.11.004.

40. Barreira, T. V., Schuna, J. M., Mire, E. F., Katzmarzyk, P. T., Chaput, J. P., Leduc, G., Tudor-Locke, C. 2015. Identifying children's nocturnal sleep using 24-h waist accelerometry. Med Sci Sports and Exerc, 47:937-943. doi: 10.1249/MSS.0000000000000486.

41. Taylor, R. W., Williams, S. M., Farmer, V. L., Taylor, B. J. 2015. The stability of sleep patterns in children 3 to 7 years of age. J Pediatr 166, 697-702 e1. doi: 10.1016/j.jpeds.2014.11.014.

42. Hjorth, M.F., Chaput, J-P., Titz, C., Dalslov, S.M., Andersen, R., Tetens, I., Michaelsen, A.F., Sjodin, A. 2014. Fatness predicts decreased physical activity and increased sedentary time, but not vice versa: Support from a longitudinal study in 8to 11-year-old children. Int J Obes 38(7):959-965. doi: 10.1038/ijo.2013.229.

43. Greever, C.J., Ahmadi, M., Sirard, J., Alhassan, S. 2017. Associations among physical activity, screen time and sleep in low socioeconomic status urban girls. Prev Med Rep 5(1): 275-278. doi: 10.1016/j.pmedr.2017.01.014

44. Katzmarzyk, P. T., Barreira, T. V., Broyles, S. T., Champagne, C. M., Chaput, J.-P., Fogelholm, M., Hu, G., Johnson, W. D., Kuriyan, R., Kurpad, A., Lambert, E. V., Maher, C., Maia, J., Matsudo, V., Olds, T., Onywera, V., Sarmiento, O. L., Standage, M., Tremblay, M. S., Tudor-Locke, C., Zhao, P., Church, T. S. 2013. The international study of childhood obesity, lifestyle and the environment (ISCOLE): Design and methods. BMC Public Health 13, 900. doi: 10.1186/1471-2458-13-900.

45. Bennett, C., de Silva-Sanigorski, A.M., Nichols, M., Bell, A.C., Swinburn, B.A. (2009). Assessing the intake of obesity-related foods and beverages in young children: comparison of a simple population survey with $24 \mathrm{hr}-$ recall. Int J Behav Nutr Phys Act 6:71. doi: 10.1186/1479-5868-6-71.

46. Birch, L.L., Fisher, J.O., Grimm-Thomas, K., Markey, C.N., Johnson, S.L. 2001. Confirmatory factor analysis of the Child Feeding Questionnaire: a measure of parent attitudes, beliefs and practices about child feeding and obesity proneness. Appetite 36:201-210. doi:10.1006/appe.2001.0398. 
47. Downing, K. L., Hinkley, T., Hesketh, K. D. 2015. Associations of Parental Rules and Socioeconomic Position With Preschool Children's Sedentary Behaviour and Screen Time. J Phys Act Health, 12:515-21. doi: 10.1123/jpah.2013-0427.

48. Hinkley, T., Salmon, J., Okely, A. D., Crawford, D. \& Hesketh, K. 2012a. The HAPPY study: development and reliability of a parent survey to assess correlates of preschool children's physical activity. J Sci Med Sport, 15:407-17. doi: 10.1016/j.jsams.2011.12.009.

49. Palfreyman, Z., Haycraft, E., Meyer, C. 2012. Development of the Parental Modelling of Eating Behaviours Scale (PARM): links with food intake among children and their mothers. Maternal and Child Nutrition 10:617-629. doi: 10.1111/j.1740-

8709.2012.00438.x

50. Gattshall, M.L., Shoup, J., Marshall, J.A., Crane, L.A., Estabrooks, P.A. 2008. Validation of a survey instrument to assess home environments for physical activity and healthy eating in overweight children. Int J Behav Nutr Phys Act 5:3. doi: $10.1186 / 1479-5868-5-3$.

51. Bohman, B., Ghaderi, A., Rasmussen, F. 2013. Psychometric Properties of a New Measure of Parental Self-Efficacy for Promoting Healthy Physical Activity and Dietary Behaviors in Children. European Journal of Psychological Assessment 29: 291-298. doi: 10.1186/s12966-016-0438-y.

52. Hayes AF. 2015. The PROCESS Macro for SPSS and SAS.

53. Australian Bureau of Statistics. 2014. Household Use of Information Technology, Australia, 2012-13. 8146.0. Canberra: Australian Bureau of Statistics. 
Table 1: Components of the Time2bHealthy Online Program

\begin{tabular}{|c|c|c|}
\hline Module & Module Content & $\begin{array}{l}\text { Guidelines informing } \\
\text { content }\end{array}$ \\
\hline Module 1 - Introduction & $\begin{array}{l}\text { General overview including goal-setting, Information on support from dietitian, } \\
\text { Information on Facebook group and link, Timetable for program, Weekly } \\
\text { planner. }\end{array}$ & \\
\hline Module 2 - Healthy Meals & $\begin{array}{l}\text { Introduction to healthy eating, How much food?, Serving sizes, Decreasing } \\
\text { sugar consumption, How to read labels, Decreasing saturated fat consumption, } \\
\text { Increasing fruit and vegetable consumption, Recipe modification, Getting the } \\
\text { balance right, Goal-setting. }\end{array}$ & $\begin{array}{l}\text { Australian Dietary } \\
\text { Guidelines [24] }\end{array}$ \\
\hline $\begin{array}{l}\text { Module } 3 \text { - Healthy Snacks } \\
\text { and Drinks }\end{array}$ & $\begin{array}{l}\text { Why healthy snacks and drinks?, Which snacks and drinks?, What snacks and } \\
\text { drinks are consumed in your house?, Healthy snacks and drinks, Choosing } \\
\text { snacks and drinks, Goal review, Goal-setting. }\end{array}$ & $\begin{array}{l}\text { Australian Dietary } \\
\text { Guidelines [24] }\end{array}$ \\
\hline $\begin{array}{l}\text { Module } 4 \text { - Physical } \\
\text { Activity }\end{array}$ & $\begin{array}{l}\text { What is physical activity?, Why do physical activity?, What physical activity is } \\
\text { happening now?, How to increase physical activity, Space, Equipment, Creative } \\
\text { outside activities, When to increase physical activity, Goal review, Goal-setting. }\end{array}$ & $\begin{array}{l}\text { Australia's Physical Activity } \\
\text { and Sedentary Behaviour } \\
\text { Guidelines for Children (0-5 } \\
\text { years) [25] }\end{array}$ \\
\hline Module 5 - Screen-time & $\begin{array}{l}\text { What is small screen recreation and how much?, why } 1 \text { hour?, How much small } \\
\text { screen recreation is happening now?, Too much small screen recreation?, If not } \\
\text { small screen recreation then what?, Let their imagination run wild, Active small } \\
\text { screen recreation, When can you decrease small screen recreation? Goal review, } \\
\text { Goal-setting. }\end{array}$ & $\begin{array}{l}\text { Australia's Physical Activity } \\
\text { and Sedentary Behaviour } \\
\text { Guidelines for Children (0-5 } \\
\text { years) [25] }\end{array}$ \\
\hline Module 6 - Sleep & $\begin{array}{l}\text { About sleep, What are my child's sleeping patterns now? How long should my } \\
\text { children be sleeping for? What can I do?, Persistence with resistance, } \\
\text { Improving sleep time, Goal review, Goal-setting }\end{array}$ & $\begin{array}{l}\text { National Sleep Foundation } \\
\text { Sleep Time Duration } \\
\text { Recommendations [26] }\end{array}$ \\
\hline
\end{tabular}


Table 2: Outcome measures collected

\begin{tabular}{|c|c|c|c|c|}
\hline Outcome measure & Method & Baseline & Post-intervention & 6-month follow-up \\
\hline \multicolumn{5}{|l|}{ Child } \\
\hline Weight status* & Body mass index & $\sqrt{ }$ & $\sqrt{ }$ & $\sqrt{ }$ \\
\hline Physical activity & Accelerometer & $\sqrt{ }$ & $\sqrt{ }$ & $\sqrt{ }$ \\
\hline \multicolumn{5}{|c|}{ Child (reported by parent) } \\
\hline Dietary Intake & $\begin{array}{l}\text { Questionnaire }[43] \text { and } \\
\text { 24-hour recall completed } \\
\text { via Easy Diet Diary app }\end{array}$ & $\sqrt{ }$ & $\sqrt{ }$ & $\sqrt{ }$ \\
\hline Sleep & $\begin{array}{l}\text { Accelerometer and } \\
\text { questionnaire [38] }\end{array}$ & $\sqrt{ }$ & $\sqrt{ }$ & $\sqrt{ }$ \\
\hline Screen-time & Questionnaire $[45,46]$ & $\sqrt{ }$ & $\sqrt{ }$ & $\sqrt{ }$ \\
\hline Demographics & Questionnaire & $\sqrt{ }$ & & \\
\hline \multicolumn{5}{|l|}{ Parent } \\
\hline Self-efficacy & Questionnaire [49] & $\sqrt{ }$ & $\sqrt{ }$ & $\sqrt{ }$ \\
\hline Parental role-modelling & $\begin{array}{l}\text { Modified questionnaire } \\
{[47,48]}\end{array}$ & $\sqrt{ }$ & $\sqrt{ }$ & $\sqrt{ }$ \\
\hline Child feeding & $\begin{array}{l}\text { Modified questionnaire } \\
\text { [44] }\end{array}$ & $\sqrt{ }$ & $\sqrt{ }$ & $\sqrt{ }$ \\
\hline Demographics & Questionnaire & $\sqrt{ }$ & & \\
\hline
\end{tabular}

*Primary outcome measure 
Figure 1: Time2bHealthy Study Design

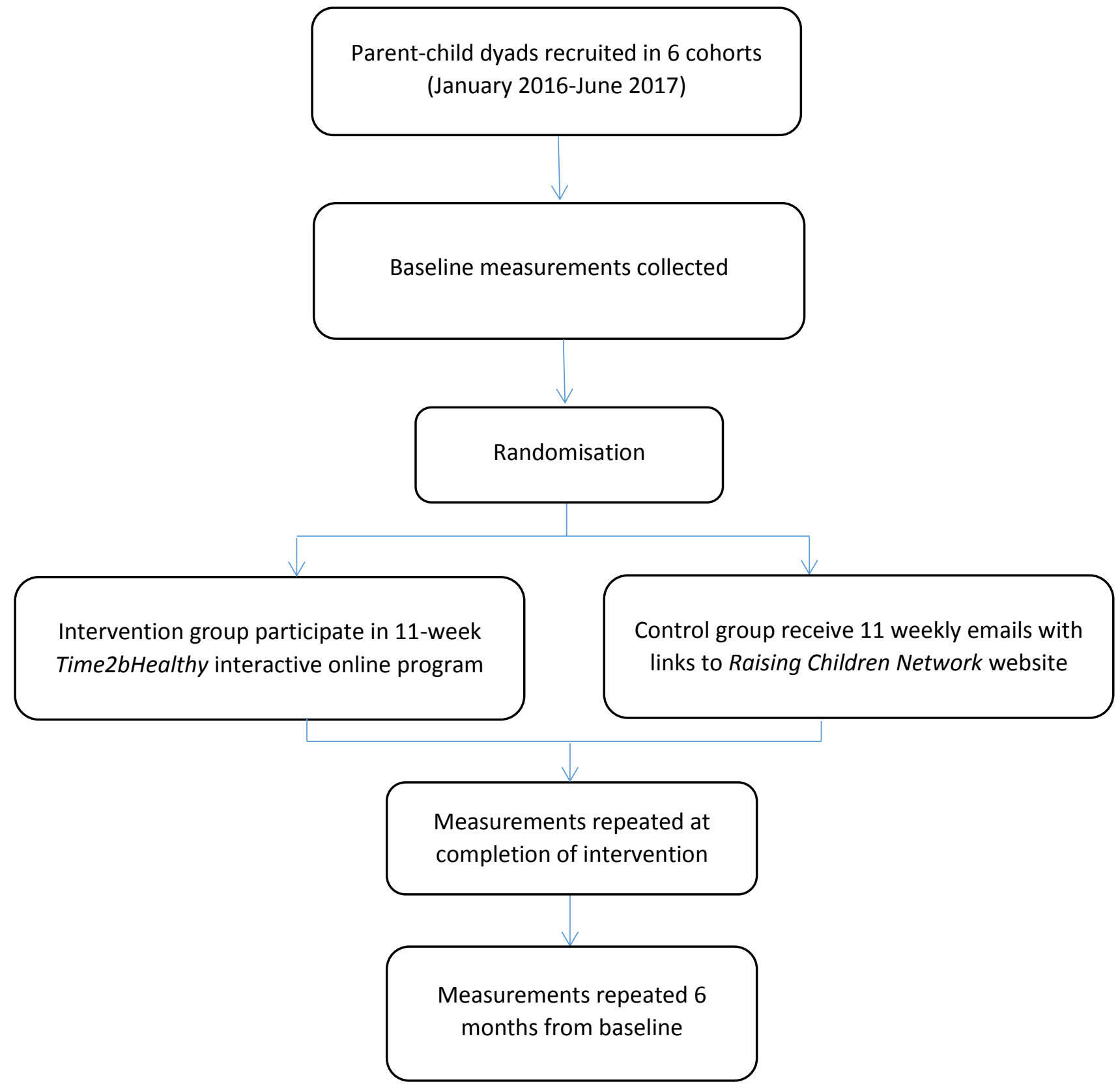


Figure 2: Personal, Environmental and Behavioural Influences Within the Time2bHealthy Program.

\section{Personal}

(knowledge of healthy eating, physical activity, screen-time and

sleep, outcome expectations, attitudes, perceived benefits and barriers, self-efficacy)
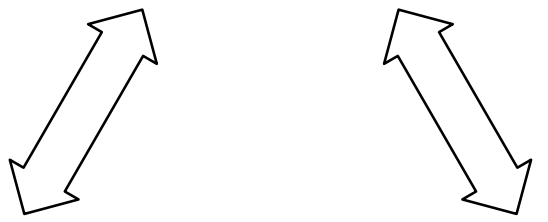

\section{Environmental}

(exposure to videos of successful implementation of skills/behaviours, communication, feedback and reinforcement from program staff and other participants)

\section{Behavioural}

(skill development, applicaion and practice of the skill through SMART goal setting and action planning, self-monitoring of progress) 
Figure 3: Backwards Intervention Mapping Process Used in the Development of the Physical Activity Module

\section{Activities}

Theory

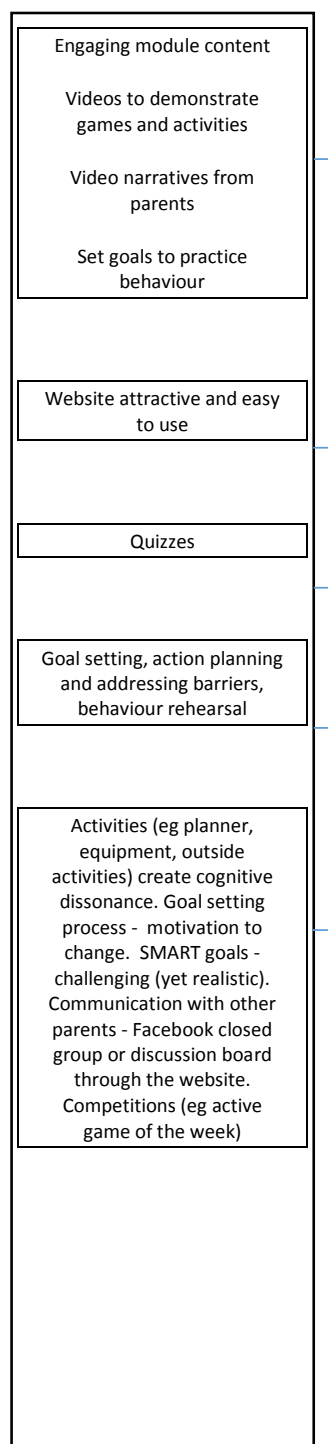

Target Behaviour

Sub Categories

Major Categories

Goal
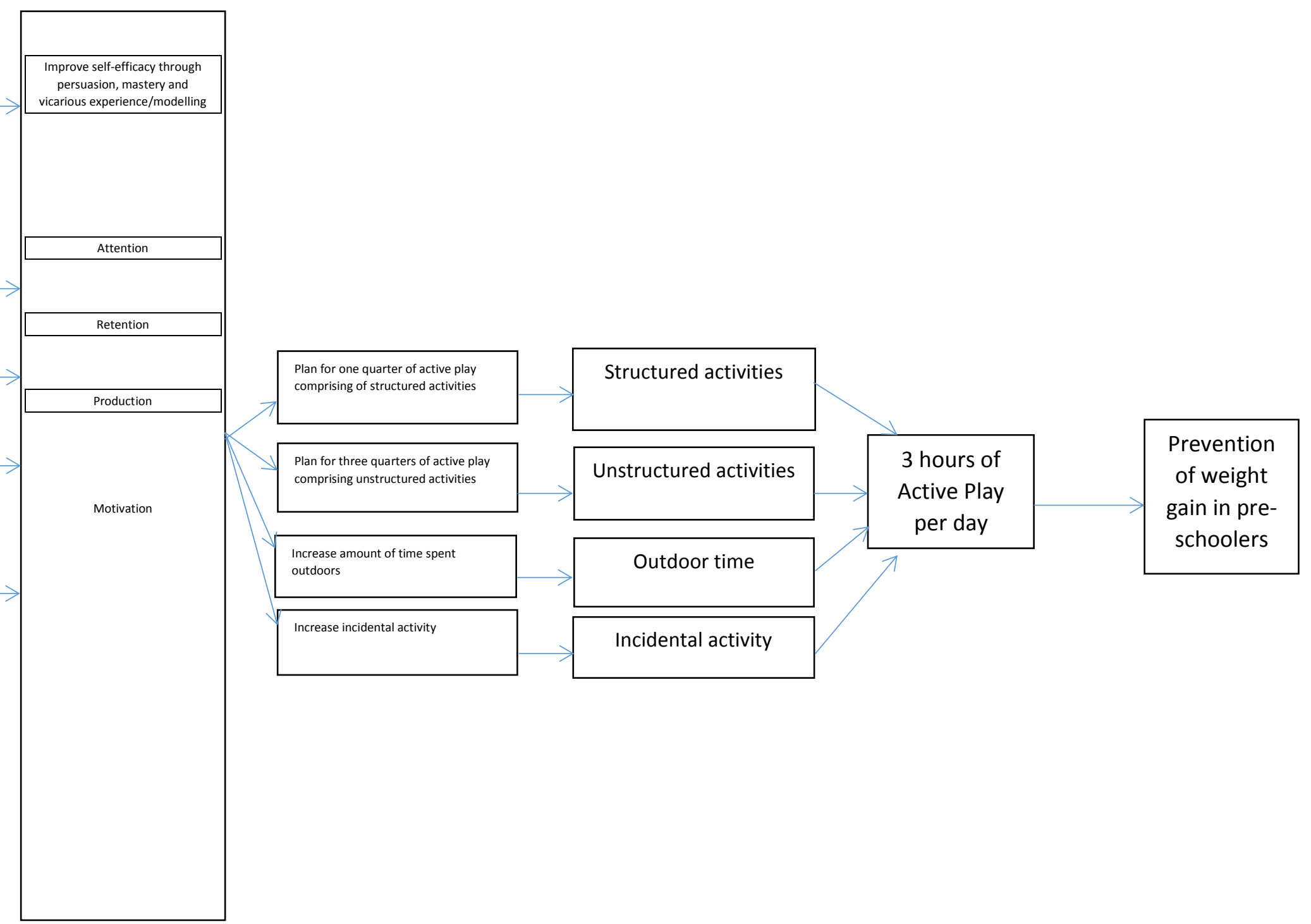
